\title{
Modelagem da Produção de Vacas Leiteiras com Utilização de Pastagem e Silagem de Milho
}

\author{
N.M. KINALSKI ${ }^{1}$, J.L. BERTO ${ }^{2}$, B.S. NETO ${ }^{3}$, Universidade Regional do Noroeste \\ do Estado do Rio Grande do Sul, Mestrado em Modelagem Matemática, Cx.P. 560, \\ 98700-000 Ijuí, RS, Brasil.
}

Resumo. Os diversos setores que compõem a cadeia de produção de leite têm debatido sobre quais sistemas de criação seriam mais viáveis para os diferentes segmentos da cadeia. A partir da programação matemática é possível lançar algumas considerações ao debate. Dessa forma, foi desenvolvido um modelo de otimização dos resultados econômicos de um rebanho de vacas em produção. Parte dos coeficientes técnicos e econômicos foram obtidos a partir de um sistema de criação e cultivo desenvolvido no Instituto Regional de Desenvolvimento Rural. Esse modelo é de programação não linear. Essa não linearidade se deve principalmente ao emprego de equações de predição da ingestão de animais em pastagens, que possuem expoentes diferentes de um. O modelo desenvolvido considera inúmeras restrições associadas às exigências nutricionais das vacas leiteiras, à disponibilidade e qualidade das pastagens e silagem e à utilização das áreas pelas diferentes culturas. $\mathrm{O}$ total de área agrícola disponibilizada para o sistema de criação e cultivo de soja foi de $72 \mathrm{ha}$. Ao se limitar o número máximo de vacas em produção em 60 , o modelo estimou o máximo local do valor agregado bruto de $R \$ 108.694,70$, alocando aproximadamente 17 ha para pastagens, 11, 3 ha e para silagem de milho e 43,1 ha de soja. Ao se liberar o número limite de vacas, o valor agregado bruto estimado atingiu um ótimo local de $R \$ 204.066,00$, com 145 vacas em produção. Nessa solução toda a área disponível foi empregada para a produção de pastagem $(41,5 h a)$ e silagem $(30,5 h a)$. De maneira geral, a produção média das vacas foi de aproximadamente 14, 9 litros, obtido principalmente pelo consumo de pastagens, porém com suplementação constante de silagem a fim de se atingir níveis mais elevados de ingestão. Em geral, os resultados estimados estão próximos aos encontrados na literatura quando se considera a produção, a ingestão e a capacidade de suporte das pastagens. Quando se compara os resultados estimados com os resultados obtidos no Instituto Regional de Desenvolvimento Rural , cujo valor agregado bruto foi de $R \$ 87.521,78$ e a produção média de leite por vaca de 17, 03 litros, é possível concluir que há uma margem considerável para atingir resultados econômicos superiores na produção de leite e nesse sentido a escolha das pastagens tem grande importância.

\footnotetext{
${ }^{1}$ n.kinalski@ibest.com.br

${ }^{2} \mathrm{DEAg} /$ UNIJUI (Orientador)

${ }^{3} \mathrm{DEAg} /$ UNIJUI (Co-orientador)
} 


\section{Introdução}

A paisagem agrícola da Região Noroeste do Estado do Rio Grande do Sul sofreu importantes transformações a partir dos anos oitenta devido às condições menos favoráveis estabelecidas para a produção de grãos, principalmente em unidades de produção agrícola familiar. De uma produção de subsistência ou de pequena participação na renda agrícola, o leite passa a ser um dos principais componentes em grande parte das unidades de produção da região.

As condições relativamente favoráveis à produção de leite passaram a sofrer modificações no decorrer dos últimos anos, de tal forma que para permanecer no processo produtivo, se tornava cada vez mais importante e necessário proceder a escolhas de combinações de fatores que possibilitassem atingir níveis de produção e qualidade do leite com custos adequados ao comportamento imposto pelo mercado. Além é necessário considerar o resultado econômico que satisfaça os objetivos de renda e trabalho da família, frente a atividades agrícolas alternativas, como a produção de soja na região do noroeste do Rio Grande do Sul.

\subsection{Material e métodos}

O modelo de programação não-lineare foi desenvolvido no software LINGO [7], com o objetivo de otimizar o resultado econômico da produção leiteira do IRDeR (Instituto Regional de Desenvolvimento Rural). Nesse modelo, foi considerado apenas o lote de vacas em produção. No processo de otimização, o modelo define a dimensão do rebanho produtivo, a área e as forragens cultivadas para alimentar o rebanho, o rendimento médio de leite por vaca durante os diferentes meses do ano e a ingestão de pasto e silagem de milho, além da área destinada para o plantio de soja. É importante destacar que o modelo considera as diferenças de preços do leite cota e extra cota e o efeito da qualidade e da disponibilidade do pasto nos diferentes meses do ano sobre a ingestão de pasto. O leite extra cota é considerado quando a quantidade de leite (média mensal) entregue ao laticínio excede a média mensal do leite entregue nos meses considerados de entressafra. Em geral esse leite em excesso recebe remuneração inferior ao leite dito cota.

Matematicamente, o modelo é constituído por uma função objetivo e um conjunto de restrições. Porém, nem todas as restrições são do tipo linear. As não linearidades são geradas por variáveis com expoente diferente de um e devido a multiplicação de variáveis dependentes durante os procedimentos adotados no modelo. O modelo de programação matemática é representado na forma de notação matricial da seguinte maneira:

\section{Função Objetivo: MAXIMIZAR $R_{M}$}

sujeito a:

$$
\begin{gathered}
A \mathrm{X}<=0 \\
B X<=\mathrm{b} \\
C(X)>=0 \\
X>=0,
\end{gathered}
$$

onde: $R_{M}$ = resultado econômico; $X=$ vetor coluna da atividade do sistema de produção; $A=$ matriz que envolve as restrições associadas à dieta dos bovinos de 
leite; $B=$ matriz onde estão envolvidas as restrições de superfície utilizadas para o manejo e produção; $b=$ é o vetor coluna das restrições relativas à superfície; $C(X)=$ conjunto de funções não-lineares (uso ou não da silagem na dieta dos bovinos de leite e as equações de ingestão).

As variáveis do vetor $b$ referem-se às restrições da superfície útil para o plantio de pastagens, atribuídas ao modelo.

\section{Descrição do Modelo}

A função objetivo procura maximizar os resultados econômicos da produção de leite considerando a quantidade e preço do leite cota e extra cota produzidos, subtraindose o custo intermediário das pastagens e silagem usadas anualmente e outros itens que compõem os demais custos intermediários associados unicamente às vacas. As variáveis associadas à produção de leite estão expressas em $\mathrm{kg}$ de leite produzido, a variável associada ao animal está expressa em número médio de vacas em produção durante todo o ano, e as variáveis associadas às pastagens e silagem estão expressas por unidade de área $(h a)$. Como alternativa para o uso da área de verão o modelo também inclui o valor agregado bruto por hectare da cultura de soja.

$$
\begin{array}{r}
M A X=0,32 * L C O T T A+0,32 * 0,8125 * L E X T-56 * V L-178 * T I F T- \\
307 * E L E F-231 * M I L T-280 * Z T-286 * A Z T-247 * A V A Z-\text { SILA+ } \\
494 * \text { SOJA. }
\end{array}
$$

Restrições: os principais grupos de restrições estão associadas ao tamanho e alocação das áreas, ao número de vacas em lactação, ao balanço de nutrientes para manutenção e produção de leite das vacas, à capacidade de ingestão do animal diante das condições das pastagens e ao manejo de suplementação alimentar.

Restrições de superfície agrícola útil: no modelo pode-se optar por limitar a área e flexibilizar o número de vacas em lactação, ou vice-versa. Quando se opta por limitar a área se deve considerar o fato de que há dois períodos de cultivo de pasto e, portanto, de uso dessas áreas. As áreas de pastagens perenes de clima quente não permanecem disponíveis para serem usadas no inverno. Já as áreas de cultivo de pastagens anuais de estação quente, silagem de milho e soja podem ser empregadas para cultivo de pastagens anuais de estação fria. A condição estabelecida é que o somatório das áreas empregadas para pastagens, silagem e grãos deve ser menor ou igual à superfície total disponível para tais atividades em cada período, e que é apresentada da seguinte forma:

$[S A U V E R B] E L E F+T I F T+M I L T+S O J A+S I L A-S A U T V<=0 ;$ $[S A U I N V B] E L E F+T I F T+Z T+A V A Z+A Z T-S A U T I<=0$.

Restrição do Número de Animais: essa restrição é gerada a partir da relação entre a ingestão total de volumosos das vacas e da quantidade global de volumosos disponíveis para o animal consumir no mês considerado. Volumosos são as pastagens e a silagem. A quantidade de volumosos que as vacas podem ingerir dependem das áreas destinadas em cada mês, ao seu rendimento e no caso específico das pastagens a um coeficiente de utilização máxima da pastagem disponível. Esse coeficiente foi 
assumido como sendo de 0,6 , ou seja, do que está disponível por área a vaca ingere até $60 \%$. Expresso da seguinte forma:

$$
\sum \operatorname{ITOTVL}_{i x} * V L=\sum \operatorname{Rend}_{i x} * \text { kcons }_{i x} * \operatorname{area}_{i x} 1000,
$$

onde $i x$ indica os meses.

A ingestão de silagem depende da estimativa de ingestão máxima da vaca, da ingestão de pasto e de um coeficiente que estima a taxa de substituição de silagem. Essa taxa de substituição foi empregada para estabelecer menor ingestão de silagem, considerando o efeito depressor que os alimentos conservados na forma de ensilagem causam na ingestão. Aqui foi empregado o valor de 1,2, ou seja, a silagem apresenta uma depressão de $20 \%$ na ingestão. E isso foi expresso na seguinte forma:

$$
I N G P A S T O_{X}+S U B S I L * I S I L V L_{X}-I N G P<=0 .
$$

Restrições nutricionais: o modelo considera o balanço de energia metabolizada $(E M)$ e proteína bruta $(P B)$ segundo [5]. Para ambos os nutrientes a vaca primeiramente satisfaz as demandas para manutenção e o que restar ela transforma em leite. Esta restrição fica:

$$
\begin{array}{r}
E N P L \sum_{i=1}^{n}\left(P_{n} V L\right)+E N M A N T * V L- \\
\sum(A R E S T A P A S T * E N P A S T . K C O N S . R P A S T) .
\end{array}
$$

Capacidade de ingestão de matéria seca: a ingestão de pasto potencial é estimada segundo o modelo descrito em [1], que é ajustada para as condições de disponibilidade ( $\mathrm{kg}$ de matéria seca de pasto por hectare) e de qualidade (coeficiente de digestibidade) da pastagem [1] e [3]. A estimativa da ingestão potencial das vacas foi obtida considerando que todas as vacas do plantel iriam permanecer em lactação por 10 meses, e a distribuição dos partos ocorreria de maneira homogênea entre os 12 meses do ano e as vacas se encontrariam em média com 150 dias de lactação. Considerou-se também que os animais pesassem $550 \mathrm{~kg}$, com idade média de 48 meses, peso ao nascer de $42 \mathrm{~kg}$, peso maduro de $700 \mathrm{~kg}$ e potencial de produção no momento de máxima produção de $35 \mathrm{~kg}$ de leite.Os coeficientes associados à qualidade e à disponibilidade da pastagem foram calculados a partir da média do coeficiente de digestão e da disponibilidade média das pastagens utilizadas pelos animais em cada mês. Esses coeficientes geram os fatores que restringem a ingestão das vacas a partir das equações citadas acima. No presente modelo, a ingestão ainda é influenciada pela possibilidade de se usar alimentação suplementar, foi assumido que a substituição é expressa por um valor de 1,4 para silagens, ou seja, cada quilograma de silagem ingerido tem um efeito sobre a ingestão equivalente a $1,4 \mathrm{~kg}$ de pasto de boa qualidade.

\section{Resultados e Discussão do Modelo}

O modelo aqui descrito foi empregado para avaliar alternativas que possibilitem atingir resultados econômicos máximos em sistemas com produção de leite. Na primeira série de simulações, foi considerada a restrição máxima de 60 vacas em 
produção. A restrição de 60 vacas em produção corresponde à capacidade máxima do curral de alimentação das vacas. Cada série de simulações foi realizada alterandose o número mínimo de vacas. Essa alteração possibilitou obter resultados de ótimos locais diferentes entre algumas simulações.

\subsection{Resultados do modelo quando houve restrição do número máximo de vacas em 60}

O valor agregado bruto máximo estimado foi de $\mathrm{R} \$ 108.694,70$ (Tabela 1). Valores próximos a esse foram obtidos com pequenas variações nas combinações, ou até mesmo considerando mudanças maiores, como a inclusão de maior área de tifton que demandou o aumento da área de silagem e com isso resultou numa redução de aproximadamente $10 \%$ no VAB. Essa diferença também se deve à produção de leite, a qual apresentou menor produção global e maior produção de leite extra cota quando a opção foi à pastagem de tifton. De maneira geral, a partir das soluções apresentadas na Tabela 1 constata-se que a restrição de um máximo de 60 vacas em produção imposta ao modelo é que permitiu a ocupação de 55cultura de soja. Os ótimos locais apresentados foram obtidos com uma combinação de pastagens de estação quente em que predominaram as pastagens perenes (aproximadamente 16 ha) de capim-elefante e/ou tifton, com nenhuma ou muito baixa participação de pastagem cultivada de estação quente (milheto). Das pastagens de estação fria, apenas a consorciação de aveia com azevém e trevo foi empregada (16 ha). A silagem esteve presente em todas as soluções e ocupou uma área mínima de 11,31 ha até o máximo de 14,71 ha, ou seja, uma diferença de 3,40 ha. Diferença esta semelhante à variação da área de soja. Com as combinações apresentadas, a superfície disponível foi totalmente utilizada no verão, enquanto que no inverno, desconsiderando-se as culturas permanentes, apenas 16ha foram utilizados, o que equivale a 30

As combinações apresentadas na Tabela 1 estimaram uma produção total de leite superior a 304 mil litros de leite no ano, até aproximadamente 330 mil. Desses, apenas $6,7 \%$ a 16,1\% foram leite extra cota. Essa condição em parte foi determinada pelo fato de se assumir que os partos ocorrem equilibradamente em todos os meses do ano, sem que haja concentrações em determinados períodos. Conseqüentemente, isso impede que se considere a possibilidade de melhor adequar os partos frente à disponibilidade de pasto.

Ao se analisar os resultados da simulação que permitiu atingir um ótimo local de $R \$ 108.697,70$, é possível observar que a ingestão de volumoso (pasto e silagem) mensal variou de 14,2 a 17,0 kg de matéria seca (Figura 1), enquanto a produção mensal de leite variou de 9,6 a 21,0 L. A primeira constatação é a de que, em momento algum, a ingestão potencial $(18,9 \mathrm{~kg} / \mathrm{d})$ foi plenamente atingida pelas vacas. Essa restrição se deve ora a disponibilidade ora à qualidade das pastagens que foram compensadas, em parte, pelo emprego de silagem (Figura 2).

Durante o verão, a ingestão de matéria seca estimada de pastagem (Figura 2) variou entre 1,0 a $2,73 \%$ do peso vivo $(P V)$ das vacas, com média de $2,2 \%$ do peso vivo, valor próximo aos encontrados na literatura [2]. Nos meses em que a oferta foi de pastagens de estação fria, a ingestão estimada de pasto variou de 1,85 a 2,7\% do PV, com média de 2,26\% do peso vivo. Nas pastagens de estação fria, a restrição 
Tabela 1: Resultados de alguns ótimos locais superiores obtidos com o modelo considerando o limite máximo de 60 vacas em lactação: valor agregado bruto, produção de leite e distribuição das áreas entre as culturas

\begin{tabular}{llllll}
\hline Simulações & $\mathrm{A}$ & $\mathrm{B}$ & $\mathrm{C}$ & $\mathrm{D}$ & $\mathrm{E}$ \\
\hline Função objetivo (VAB R\$) & 108694,70 & 108085,40 & 99903,66 & 98626,63 & 98394,25 \\
Número vacas & 60,00 & 60,00 & 60,00 & 60,00 & 60,00 \\
$\begin{array}{l}\text { Produção total } \\
\text { de leite (L/ano) }\end{array}$ & 328418,70 & 330320,80 & 307210,20 & 304183,80 & 304719,80 \\
Produção diária de & 14,99 & 15,08 & 14,03 & 13,88 & 13,91 \\
leite por vaca (L/VL/d) & & & & \\
Leite cota (L) & 306534,70 & 307873,00 & 263040,40 & 264037,80 & 255661,90 \\
Leite extra cota (L) & 21884,00 & 22447,83 & 44169,80 & 40145,99 & 49057,87 \\
Tifton (ha) & 5,63 & 1,70 & 15,64 & 15,37 & 15,64 \\
Elefante (ha) & 11,96 & 15,62 & 0,30 & 0,00 & 0,30 \\
Azevém trevo (ha) & 0,00 & 0,32 & 0,00 & 0,00 & 0,00 \\
Aveia azevém trevo (ha) & 16,19 & 16,19 & 15,55 & 16,19 & 16,19 \\
Silagem de planta & 11,31 & 12,27 & 14,25 & 14,71 & 14,54 \\
inteira de milho (ha) & & & & & 40,12 \\
Soja (ha) & 43,11 & 42,40 & 40,40 & 39,94 & 4 \\
\hline Fonte: Resultados obtidos da simulação, variando-se o número mínimo de vacas.
\end{tabular}

Fonte: Resultados obtidos da simulação, variando-se o número mínimo de vacas.

da ingestão foi significativamente influenciada pela disponibilidade de pasto.

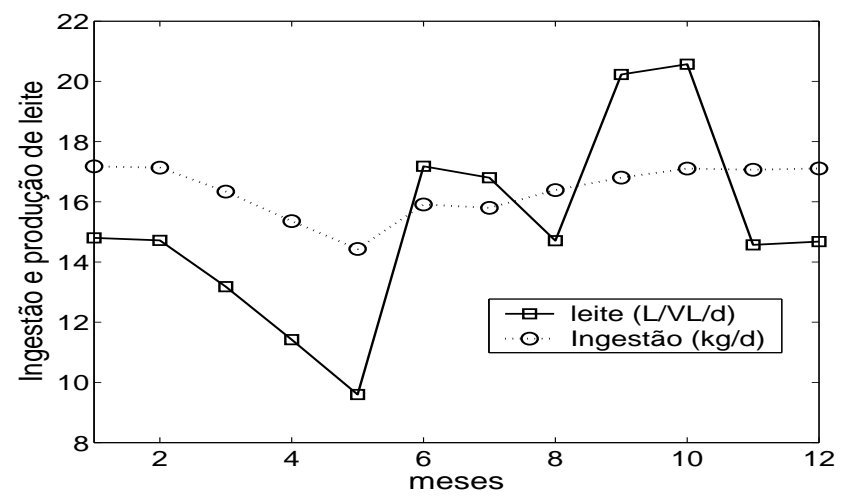

Figura 1: Ingestão estimada de matéria seca de volumoso $(\mathrm{kg} / \mathrm{d})$ e produção de leite diária $(L / V L / d)$.

A essa ingestão de pasto o modelo adicionou a ingestão de silagem, o que possibilitou atingir ingestões superiores em todos os meses. A ingestão de silagem foi maior nos meses de abril a julho, chegando a representar mais de $60 \%$ da dieta das vacas em maio e apenas $12 \%$ de setembro a fevereiro. Com isso, a produção de leite estimada variou de um mínimo de $9,6 L / V L / d$, em maio, até um máximo de aproximadamente $21 L / V L / d$ nos meses de setembro e outubro. A produção de leite estimada nos meses em que houve consumo de pastagem de estação quente (novem- 


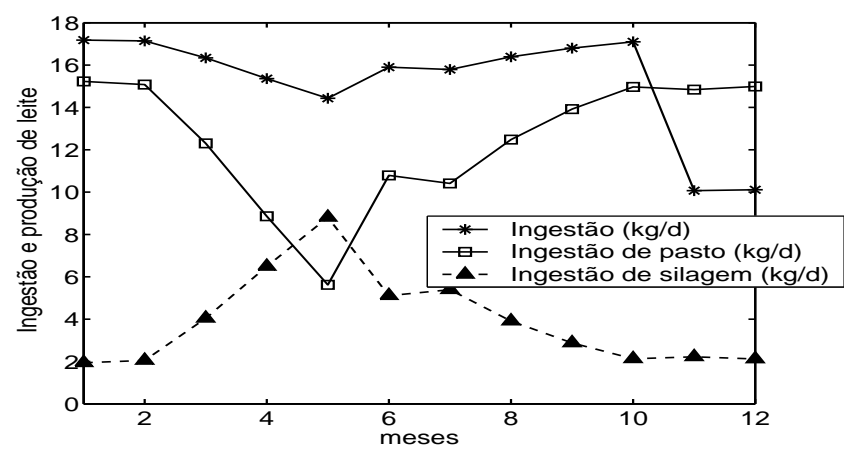

Figura 2: Ingestão de $M S$ potencial de silagem e de pasto durante o ano.

bro a abril) variou de 12 a $15 \mathrm{~L}$, sendo menor nos meses de menor disponibilidade de pasto (março e abril). Já nos meses em que as pastagens de clima temperado estiveram disponíveis (junho a outubro), a produção de leite estimada variou de 16 a $22 \mathrm{~L}$. A distribuição de silagem foi realizada em todos os dias do ano (Figura 2). Nos meses de maior oferta de pastagem, a inclusão foi reduzida (próximo a $2 \mathrm{~kg}$ de $M S$ ), chegando a atingir um consumo de $9 \mathrm{~kg}$ em maio, quando a oferta de pasto foi muito baixa. A adição da silagem ao consumo de pasto em vários meses se deve ao fato de que o animal tem um custo nutricional para se manter e dessa forma a ingestão de silagem representa um ganho marginal importante ao garantir a ingestão de uma maior quantidade relativa de nutrientes que se destinam à produção de leite.

\subsection{Resultados do modelo sem restrição para o número má- ximo de vacas}

A Tabela 2 apresenta alguns dos resultados obtidos a partir do modelo sem restrição para o número de vacas em lactação. O máximo local estimado para o valor agregado bruto foi de $R \$ 204.066,30$ (Tabela 2), aproximadamente $R \$ 96.400,00$ superior ao $V A B$ máximo estimado com a restrição de vacas. A produção leite total ficou em torno de $781 \mathrm{milL} /$ ano. Desse leite produzido, apenas $10 \%$ foi de leite extra cota. De maneira geral, constata-se que o número de animais em produção foi estimado em aproximadamente 145 vacas e $100 \%$ da área foi destinada para a alimentação das vacas em lactação, em detrimento do cultivo de soja. A combinação de forrageira na estação quente do ano foi de aproximadamente $22 \mathrm{ha}$ de pastagens perenes, predominando o capim-elefante, com mais 9 ha de milheto e 30, 5ha de milho para silagem. Na estação fria, a escolha predominante foi por 39 ha da mistura de aveia, azevém e trevo. Dessa forma, na estação quente toda a área considerada foi empregada para o cultivo de forrageiras, enquanto na estação fria, das áreas livres $(50 h a)$, menos de $80 \%$ foi empregada para a produção de forragens.

Essa combinação de forragens possibilitou uma ingestão média pouco superior a $17 \mathrm{~kg}$ de MS por vaca diariamente e com isso uma produção média de aproximada- 
Tabela 2: Valores estimados pelo modelo quando não se limitou o número de vacas em lactação - valor agregado bruto (ótimo local), distribuição das culturas e produção de leite

\begin{tabular}{ll}
\hline Variável & Valor \\
\hline Função objetivo (VAB R $\$$ ) & 204066,30 \\
Produção de leite total & 781034,88 \\
Produção de leite por dia & 14,78 \\
Leite cota & 700451,20 \\
Leite extra cota & 80583,68 \\
Vacas Lactação & 144,84 \\
Tifton & 3,80 \\
Elefante & 28,60 \\
Milheto & 9,07 \\
Azevém trevo & 0,52 \\
Avia azevém trevo & 39,09 \\
Silagem & 30,53 \\
\hline \multicolumn{2}{l}{ Fonte: resultado de uma simulação do programa de otimização }
\end{tabular}

mente $14,8 \mathrm{~L} / \mathrm{VL} / \mathrm{d}$. Valores muito semelhantes aos estimados quando o modelo foi executado com restrição de 60 vacas em produção (Tabela 1). Da mesma forma, no mês de maio percebe-se uma baixa ingestão e, conseqüentemente, uma baixa produção de leite (Figura 3), pois nesse período os animais ingerem apenas silagem em detrimento do uso de pasto devido à sua baixa disponibilidade. A variação da produção de leite por vaca também foi semelhante ao comportamento apresentado nos resultados quando o máximo de vacas ficou restrito a 60.

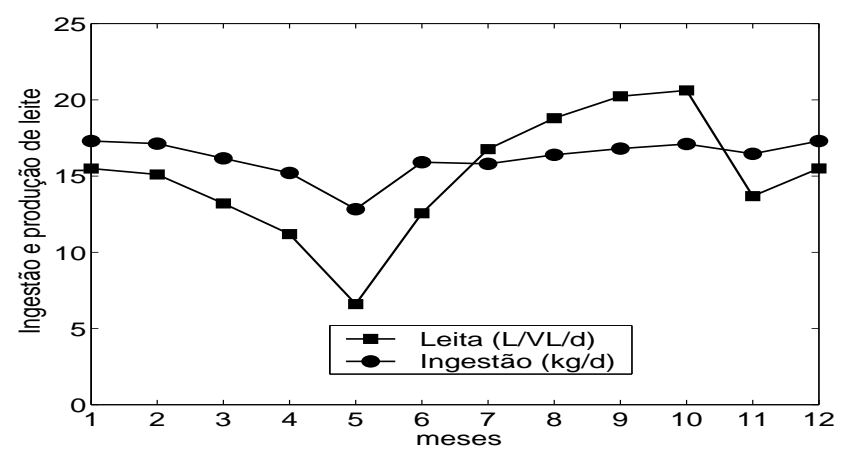

Figura 3: Ingestão de $M S$ de volumoso $(k g / d)$ e produção de leite $(L / V L / d)$

Comparação dos resultados estimados pelo modelo e os resultados obtidos no IRDeR

A comparação dos resultados da simulação e os resultados obtidos no IRDeR 
apresentados na Tabela 3, permitem observar que há uma grande diferença do VAB obtido e do VAB estimado pelo modelo. Isso permite supor que há uma margem de manobra para melhorar o desempenho econômico da atividade leiteira no IRDeR.

Tabela 3: Comparação entre os dois sistemas de produção

\begin{tabular}{rrr}
\hline Sistemas & $\begin{array}{r}\text { IRDeR } \\
\text { (60 vacas) }\end{array}$ & $\begin{array}{r}\text { Modelo } \\
\text { (60 vacas) }\end{array}$ \\
\hline Valor agregado bruto & $87.521,78$ & $108.694,70$ \\
Produção de leite/ dia & 17,03 & 14,99 \\
Litros de leite (ano) & $373.048,00$ & $328.418,07$ \\
Concentrado (toneladas) & 137.19 & 0 \\
Milho silagem (há) & 14,00 & 11,305 \\
Aveia azevém (ha) & 3,64 & 0 \\
Azevém trevo (ha) & 9,10 & 0 \\
Mveia, azevém e trevo (ha) & 14,76 & 16,2 \\
Milheto (ha) & 15,04 & 0 \\
Capim elefante(ha) & 5,28 & 11,956 \\
Tifton (ha) & 5,67 & 5,626 \\
Área disponível & 72,00 & 72 \\
\hline Fonte: Dados simulados pelo modelo $(2004)$ e obtidos no IRDeR (2002).
\end{tabular}

\section{Conclusão}

A solução encontrada no modelo permite observar que a margem de melhora no $V A B$ pode ser elevada, isso segundo as condições observadas e estimadas no IRDeR. Essa margem de melhora do desempenho econômico se relaciona mais com a redução de custos de produção do leite do que com o aumento da produção de leite global ou produtividade de leite por animal, sugerindo melhor adequação dos recursos empregados.

O modelo indica que as pastagens que apresentam ciclo mais longos de oferta de forragem para os animais são preferenciais para comporem a combinação de culturas do sistema que maximizam o $V A B$.

Os resultados indicaram que em todos os meses as vacas encontram restrição à ingestão de pasto e que o uso de silagem possibilitou atingir níveis mais elevados de ingestão e produção de leite, sendo uma alternativa economicamente viável para otimizar os resultados econômicos quando empregada como suplemento.

A inclusão de concentrados feita na dieta das vacas no IRDeR tem permitido maior produção por animal, em relação aos resultados da simulação. Contudo, parece ser possível maximizar os resultados econômicos sem atingir níveis de produção por animal elevados. O presente modelo é, portanto, parte inicial de um esforço de construir modelos que permitam auxiliar a tomada de decisão nos processos de produção agrícola. Dessa forma, a partir dessa etapa pode-se sugerir que nos trabalhos futuros se procure reduzir as não linearidades do modelo e se incluir 
fatores, como suplementos concentrados, a possibilidade de variar a época de parto das vacas e outras categorias do sistema de criação para permitir uma avaliação mais ampla das atividades desenvolvidas nos sistemas de criação e produção. Além disso, é necessário um refinamento nos coeficientes técnicos. Inicialmente isso poderia ser obtido com um processo de acompanhamento mais detalhado do sistema de criação e do sistema forrageiro.

\section{LISTA DE SÍMBOLOS}

$\begin{array}{ll}\text { AVAZ } & \text { área consorciada de aveia com azevém (ha) } \\ \text { AZT } & \text { área consorciada de aveia, azevém e trevo vesiculoso (ha) } \\ \text { ELEF } & \text { área de capim-elefante (ha) } \\ \text { LCOTTA } & \text { total de leite cota produzido durante o ano (L) } \\ \text { LEXT } & \text { leite extra cota (L) } \\ \text { MILT } & \text { área total do milheto (ha) } \\ \text { SILA } & \text { área cultivada de milho para silagem (ha) } \\ \text { SOJA } & \text { área cultivada com soja (ha) } \\ \text { SAUI } & \text { superfície agrícola útil no inverno (ha) } \\ \text { SAUV } & \text { superfície agrícola útil no verão (ha) } \\ \text { TIFT } & \text { área total de tifton (ha) } \\ \text { VL } & \text { número de vacas em lactação } \\ \text { ZT } & \text { área do consórcio de azevém com trevo (ha) } \\ \text { KCONS } & \text { proporção do pasto que é ingerido do consumido } \\ \text { PnVL } & \text { produção no mês n das vacas em lactação (L) } \\ \text { ITOTVL } & \text { ingestão total mensal das vacas em lactação (kg) } \\ \text { ENMANT } & \text { energia para mantença das vacas em produção (Mcal/dia) } \\ \text { Rend } d_{i} x & \text { rendimento do pasto em um deteminado mês } \\ \text { area } x & \text { área de pastegem em um determinado mês } \\ S I L A V L_{x} & \text { area de sialgem consumida em um determinado mês } \\ I N G P A S T O_{x} & \text { ingestão de pasto em um determinado mês do ano } \\ \text { SUBSIL } & \text { taxa de substituição de silagem } \\ \text { ISILV } L_{x} & \text { ingestão de silagem (kg/animal/mês) em um determinado mês } \\ \text { INGP } & \text { ingestão potencial (kg/animal/mês) } \\ \text { AREAPAST } & \text { área destinada a pastagem } \\ \text { VAB } & \text { valor agregado bruto }\end{array}$

Abstract. The several sectors that compose the chain of milk production have debated on which creation systems would be more feasible for the different segments of the chain. From the mathematical programming it's possible to throw up some considerations to the debate. So an optimization model of the economic results of a herd of cows in production was developed. Part of the economic and technical coefficients had been gotten from a creation and cultivation system developed in the Regional Institute of Rural Development. It is a nonlinear programming model. This non-linearity is mainly due to the use of prediction equations of the ingestion of animals in pastures, which possess exponents different from one. The developed model considers innumerable restrictions associated with the nutritional demands of dairy cows, with the availability and quality of pastures and ensilage, and with the utilization of the areas by the different cultivations. The total agricultural area available for the creation and cultivation system of soy was 72 hectares. When it 
is limited the maximum number of cows in production in 60 , the model has estimated the maximum point of gross added value in $R \$ 108,694.70$, allocating about 17 hectares for pastures, 11.3 hectares for maize ensilage and 43.1 hectares for soy. When it's released the limit number of cows, the estimated gross added value reached an excellent point of $R \$ 204,066.00$, with 145 cows in production. In this solution all the available area was used for the pasture production ( 41.5 hectares) and ensilage (30.5 hectares). In general way, the cows' average production was about 14.9 liters, which has been obtained mainly by the pasture consumption, however with constant supplementation of ensilage in order to reach higher levels of ingestion. In general, the estimated results are close to the ones found in literature, when it's considered the production, the ingestion and the support capacity of pastures. When it's compared the estimated results with the ones obtained in the Regional Institute of Rural Development, whose gross added value was $R \$ 64,309.84$ and the average milk production was 17.03 liters per cow, it's possible to conclude that there is a considerable margin to reach superior economic results in milk production and in this way, the choice of pastures has great importance.

\section{Referências}

[1] Australian Agricultural Council (AAC), "Feeding Standards for australian Livestock", Ruminants, CSIRO, Victoria, 1994.

[2] L.J.M. Aroeira, Estimativa de consumo de gramíneas tropicais, em "Simpósio Internacional de Digestibilidade em Ruminantes", Anais pp. 127-164, UFLAFAEPE, Lavras, 1997.

[3] J.L. Berto, "Avaliação de um modelo de predição do consumo de Capim Elefante por vacas leiteiras", Tese de doutorado, Universidade Federal.

[4] D.J. Hulme, R.C. Kellaway, P.J. Booth, et al., The CANDAIRY model for formulating and analyzing dairy cow rations, Agricultural Systems, Esses, 22, No. 2 (1986), 81-108.

[5] National Research Council (NRC): "Nutrient Requirements of Dairy Catle", National Academy of Sciences, Washington D.C., 1989.

[6] C.A. Rotz, J.R. Black, D.R. Mertens e D.R. Buckmaster, DAFOSYM. A model of the dairy forage system, Journal of Production Agriculture, 2, No. 1 (1989), 83-91.

[7] L. Schrage, "Otimization Modeling With LINGO", LINDO Sytems Inc., Chigago, 1998.

[8] J.T. Scott Jr. e E.E. Broadbent, A computerized cattle feeding program for replacent and rotain formulation, Illinois Agricultural Economics, 12, (1972). 
